\title{
INDEX OF AN IMPLICIT DIFFERENTIAL EQUATION
}

\author{
L. S. Challapa and M. A. S. Ruas
}

Abstract

In this paper we introduce the concept of the index of an implicit differential equation $F(x, y, p)=0$, where $F$ is a smooth function, $p=\frac{d y}{d x}, F_{p}=0$ and $F_{p p}=0$ at an isolated singular point. We also apply the results to study the geometry of surfaces in $\mathbb{R}^{5}$.

\section{Introduction}

Let $F(x, y, p)=0$ be an implicit differential equation (IDE), where $F$ is a smooth function and $p=\frac{d y}{d x}$. If $F_{p}\left(q_{0}\right) \neq 0$ at $q_{0} \in \mathbb{R}^{3}$, the IDE can be written locally in the form $p=g(x, y)$ and studied using methods from the theory of ordinary differential equations. When $F_{p}\left(q_{0}\right)=0$, the equation may define locally more than one direction in the plane. The cases that have been most intensively studied are the IDE's that define at most two directions in the plane. This is the case, for example, when:

$$
F(x, y, p)=0, \quad F\left(q_{0}\right)=F_{p}\left(q_{0}\right)=0, \quad F_{p p}\left(q_{0}\right) \neq 0 .
$$

A natural way to study these equations is to lift the multi-valued direction field determined by the IDE to a single field $\xi$ on the surface $M=F^{-1}(0)$. (This field is determined by the restriction to the manifold of the contact planes associated with the standard contact form $d y-p d x$ in $\mathbb{R}^{3}$.) If 0 is a regular value of $F$, then $M$ is smooth and the singularity of the projection to the plane is, generically, a fold or cusp. The critical set of this projection is called the criminant and its image is the discriminant of the equation.

In [9], Davydov classified (following the work of Dara [8]) generic bi-valued fields when the discriminant is smooth and showed that the topological normal form of the IDE acquires moduli when the discriminant is a cusp.

2000 Mathematics Subject Classification. Primary: 34A09; Secondary: 34A26, $58 \mathrm{~K} 05$.

Key words. Index, quadratic differential form, binary differential equation. The first author was supported in part by FAPESP Grant 06/50075-3. 
Implicit differential equations have extensive applications to differential geometry of surfaces, partial differential equations, control theory and singularity theory. For example, lines of curvature, asymptotic and characteristic lines on a smooth surface in $\mathbb{R}^{3}$ are given by IDE's [4] and the characteristic lines of a general linear second-order differential equation are also given by an IDE [15].

Bruce and Tari introduced in [3] the multiplicity of an IDE, at a singular point, as the maximum number of singular points of the implicit differential equation which emerge when perturbing the equation $F$. In $[\mathbf{5}]$ and $[\mathbf{6}]$ the first author defined the index of an IDE (1) in terms of generic perturbations of the IDE and showed that this index is independent of the choice of a generic perturbation. A formula that expresses the index in terms of the gradient of $F$ and the index of the 1 -form $d y-p d x$, defined on a surface with isolated singularities $M$ was also obtained. One of the main results in $[5]$ and $[\mathbf{6}]$ is the invariance of the index by smooth equivalences.

In this work we define the index of an IDE at an isolated singular point at which $F_{p p}\left(q_{0}\right)=0$. This definition extends the definition of the index of an IDE given in [5] and [6]. We also apply the results to study the geometry of surfaces in $\mathbb{R}^{5}$.

\section{Implicit differential equations}

As mentioned in the Introduction, an implicit differential equation is of the form

$$
F(x, y, p)=0,
$$

where $p=\frac{d y}{d x}$ and $F$ is a smooth function in $\mathbb{R}^{3}$. An integral curve of the IDE (2) is a smooth curve $\alpha=\left(\alpha_{1}, \alpha_{2}\right):(-1,1) \rightarrow \mathbb{R}^{2}$ such that $\alpha_{1}^{\prime}(t) \neq 0$ and $F\left(\alpha(t), \frac{\alpha_{2}^{\prime}(t)}{\alpha_{1}^{\prime}(t)}\right)=0$.

Consider the surface $M=F^{-1}(0)$, and the projection $\pi: M \rightarrow \mathbb{R}^{2}$, given by $\pi(x, y, p)=(x, y)$. Generically $M$ is a smooth surface and the projection $\pi$ is generically a submersion or has a singularity of type fold, cusp or two transverse folds. The critical set $F=F_{p}=0$ of this projection is called the criminant and its image is the discriminant of the IDE.

The multi-valued direction field in the plane determined by the IDE lifts to a single vector field tangent to $M$ given by

$$
\xi=F_{p} \frac{\partial}{\partial x}+p F_{p} \frac{\partial}{\partial y}-\left(F_{x}+p F_{y}\right) \frac{\partial}{\partial p} .
$$


Equivalently, this vector field is determined by the restriction of the canonical 1-form $d y-p d x$ to the surface $M$. Note that the vector field $\xi$ may generically have an elementary zero, it is of type saddle, node or focus. One of the properties of this vector field is that the image by the projection $\pi$, of the integral curves of $\xi$ on $M$, corresponds to integral curves of the IDE.

We denote by $C_{F}$ the criminant set of the IDE. We also denote by $\omega_{\left.\right|_{C_{F}}}$ the restriction of the 1 -form $\omega=d y-p d x$ on $C_{F}$.

Definition 2.1. We say that $z_{0} \in \mathbb{R}^{2}$ is a singular point of the IDE (2) if there exists $p_{0} \in \mathbb{R}$ such that $q_{0}=\left(z_{0}, p_{0}\right)$ is a zero of the 1 -form $\omega_{\left.\right|_{C_{F}}}$.

It is easy to verify that this definition reduces to Definition 2.3 given in [5] when the IDE defines at most two directions in the plane.

Proposition $2.2([3])$. Let $q_{0} \in \mathbb{R}^{3}$ be a point on the criminant. Then $q_{0}$ is a zero of the 1-form $\omega_{\left.\right|_{C_{F}}}$ if and only if $q_{0}$ is a zero of the vector field $\xi$ or $F_{p p}\left(q_{0}\right)=0$.

From Proposition 2.2 it follows that the singular points of the IDE correspond to zeros of the vector field $\xi$ or cusps of the natural projection $\pi$. We denote by $\left(F, z_{0}\right)$ the germ of the IDE (2) at an isolated singular point $z_{0}$.

Definition 2.3. We say that $\left(F, z_{1}\right)$ and $\left(G, z_{2}\right)$ are equivalent (resp. topologically equivalent) if there exists a germ of diffeomorphism (resp. homeomorphism) $h:\left(\mathbb{R}^{2}, z_{2}\right) \rightarrow\left(\mathbb{R}^{2}, z_{1}\right)$ that sends integral curves of $\left(G, z_{2}\right)$ to integral curves of $\left(F, z_{1}\right)$.

If $q_{0}=\left(z_{0}, p_{0}\right)$ is a fold singularity of the projection $\pi$ and is an elementary zero of $\xi$, then $\left(F, z_{0}\right)$ is topologically equivalent to a well folded singularity $\left(p^{2}-y+\lambda x^{2}, 0\right), \lambda \neq 0, \frac{1}{4}$. We have a well folded saddle if $\lambda<0$, well folded node if $0<\lambda<\frac{1}{4}$ and a well folded focus if $\lambda>\frac{1}{4}$ (see $[\mathbf{9}]$ ). When $\pi$ has a cusp singularity at $q_{0}$, the equation has functional moduli with respect to topological equivalence $[\mathbf{9}]$.

Definition 2.4. ([8]) Let $q_{0} \in \mathbb{R}^{3}$ be a point on the criminant, such that $F_{p p}\left(q_{0}\right)=0$ and $F_{p p p}\left(q_{0}\right) F_{x}\left(q_{0}\right) \neq 0$.

(i) We say that $q_{0}$ is an elliptic cusp if $\left(F_{x} F_{p y}-F_{y} F_{p x}\right)\left(q_{0}\right)>0$.

(ii) We say that $q_{0}$ is a hyperbolic cusp if $\left(F_{x} F_{p y}-F_{y} F_{p x}\right)\left(q_{0}\right)<0$.

In [8], Dara studied singularities of the IDE's, describing the singularities appearing in an open and dense set in the space of all functions $F$ with the Whitney $C^{3}$-topology. The generic singularities described by 
Dara are the well folded saddle, well folded node, well folded focus and the elliptical and hyperbolic cusp.

A particular class of implicit differential equations are the binary differential equations (BDE's) of degree $n$, that is differential equations of the form

$$
a_{0}(x, y) d y^{n}+a_{1}(x, y) d y^{n-1} d x+\cdots+a_{n}(x, y) d x^{n}=0,
$$

where $a_{i}$ are smooth functions defined on $U \subset \mathbb{R}^{2}$. If $d x=0$ is not a solution of the equation (3), we can set $p=\frac{d y}{d x}$ and reduce (3) to the IDE

$$
F(x, y, p)=a_{0}(x, y) p^{n}+a_{1}(x, y) p^{n-1}+\cdots+a_{n}(x, y)=0 .
$$

We say that the IDE of degree $n$ given by (4) is totally real if $a_{i}(0,0)=0$ (for any $i=0,1, \ldots, n)$ and for all $(x, y) \in U,(x, y) \neq 0$, the equation (3) has exactly $n$ different integral curves. An IDE of degree 1 is always totally real. In the case $n=2$, an IDE is totally real if it is positive in the sense of [14]. In [12], Fukui and Nuño-Ballesteros introduce the concept of index for totally real IDE and produced a classification of generic singularities of this type of equations. Also, a generalization of the Poincaré-Hopf theorem and the Bendixon formula is obtained in [12].

In [6] the first author defined the index of an IDE of degree 2 not necessarily totally real. One of the main results in $[6]$ is the invariance of the index by smooth equivalences. We set $\delta=a_{1}^{2}-4 a_{0} a_{2}$.

Theorem $2.5([6])$. Let $(F, 0)$ be the germ of an IDE of degree 2 . If 0 is an isolated zero of the map $\left(\delta, a_{0} \delta_{x}-a_{1} \delta_{y}\right)$ and $\left(\delta, \delta_{y}\right)$, then the index of $(F, 0)$ at 0 is given by

$$
\begin{aligned}
I(F, 0)=\frac{1}{2} \operatorname{Ind}_{0}\left(\delta,\left(a_{0} \delta_{x}-a_{1} \delta_{y}\right) a_{0} \delta_{y}\right) & -\frac{1}{2} \operatorname{Ind}_{0}\left(a_{0}, a_{1}\right) \\
& -\frac{1}{2} \operatorname{Ind}_{0}\left(\delta \delta_{x}, \delta_{y}\right)+\frac{1}{2} \operatorname{Ind}_{0} \nabla \delta .
\end{aligned}
$$

When $F_{p p}(0) \neq 0$, the formula of the index simplifies. We denote by $\operatorname{Ind}_{q_{0}} \xi$ the index of the vector field $\xi$ at $q_{0} \in M$, introduced by W. Ebeling and S. M. Gusern-Zade in [10].

Theorem 2.6 $([6])$. Let $(F, 0)$ be the germ of an IDE of degree 2 , and let 0 be a zero of the vector field $\xi$. If $F_{p p}(0) \neq 0$, then

$$
I(F, 0)=\frac{1}{2}\left[\operatorname{Ind}_{0} \xi+\operatorname{Ind}_{0}\left(F_{p p} F_{y}, F_{p}, F_{x}+p F_{y}\right)\right] .
$$


A 1-parameter perturbation $F^{t}$ of the IDE (4) is determined by the 1-parameter smooth perturbations $a_{t}^{i}(x, y)=\tilde{a}_{i}(x, y, t)$ (for any $i=$ $0,1, \ldots, n)$ of its coefficients.

Definition 2.7. We say that $F^{t}$ is a good perturbation of $(F, 0)$ if all the singular points of $F^{t}$ are well folded singularities, or elliptical or hyperbolic cusps, for $t \neq 0$ sufficiently close to zero.

\section{Index of an implicit differential equation}

We refer to [16] for the basic properties of indices of vector fields used in this section.

Definition 3.1. We say that $z_{0}$ is a non-degenerate singular point of the IDE $(2)$ if $\left(F, z_{0}\right)$ is topologically equivalent to a well folded singularity, an elliptic cusp or a hyperbolic cusp.

If $z_{0}$ is a non-degenerate singular point of the $\operatorname{IDE}(2)$, then there exists $p_{0} \in \mathbb{R}$ such that $\left(z_{0}, p_{0}\right)$ is a saddle, node or focus of the vector field $\xi$ or elliptic cusp or hyperbolic cusp. So we can associate a number $K_{F}\left(z_{0}\right)$ to each non-degenerate singular point $z_{0}$ of the IDE (2), as follows:

(i) $K_{F}\left(z_{0}\right)=-1$ if $\left(z_{0}, p_{0}\right)$ is a saddle of the vector field $\xi$.

(ii) $K_{F}\left(z_{0}\right)=1$ if $\left(z_{0}, p_{0}\right)$ is a node or focus of the vector field $\xi$.

(iii) $K_{F}\left(z_{0}\right)=1$ if $\left(z_{0}, p_{0}\right)$ is an elliptic cusp.

(iv) $K_{F}\left(z_{0}\right)=-1$ if $\left(z_{0}, p_{0}\right)$ is a hyperbolic cusp.

Let $(F, 0)$ be the germ of an IDE of degree 3 given by

$$
F(x, y, p)=a(x, y) p^{3}+b(x, y) p^{2}+c(x, y) p+d(x, y)=0,
$$

such that $F(0)=F_{p}(0)=F_{p p}(0)=0$. Using equation (5) we deduce that

$$
\begin{aligned}
27 a^{2} F & =[3 a p+b]^{3}+9 a p\left[3 a c-b^{2}\right]+27 a^{2} d-b^{3} \\
3 a F_{p} & =[3 a p+b]^{2}+3 a c-b^{2} \\
F_{p p} & =2[3 a p+b] .
\end{aligned}
$$

From the above equation, we obtain that the discriminant of the IDE (5) is given by

$$
\delta=\left(27 a^{2} d-9 a b c+2 b^{3}\right)^{2}+4\left(3 a c-b^{2}\right)^{3} .
$$


Remark 3.2. If 0 is an elliptic or a hyperbolic cusp of the germ $(F, 0)$, then

$$
\operatorname{Ind}_{0}\left(F_{p p p} F, F_{p}, F_{p p}\right)=\operatorname{Ind}_{0}\left(9 a d-b c, 3 a c-b^{2}\right)=K_{F}(0) .
$$

Let $F^{t}$ be a 1-parameter perturbation of the IDE (5) given by

$$
F^{t}(x, y, p)=a_{t}(x, y) p^{3}+b_{t}(x, y) p^{2}+c_{t}(x, y) p+d_{t}(x, y)=0 .
$$

To show that $F^{t}$ is a good perturbation of the IDE (5), it is sufficient to prove that 0 is a regular value of the map $\left(9 a_{t} d_{t}-b_{t} c_{t}, 3 a_{t} c_{t}-b_{t}^{2}\right)$, for all $t \neq 0$. We denote by $\mathcal{P}_{k}\left(\mathbb{R}^{2}\right)$ the set of all polynomials of 2 variables and degree less than or equal to $k$. Let $\Phi: \mathbb{R}^{2} \times \mathcal{P}_{k}^{4}\left(\mathbb{R}^{2}\right) \rightarrow \mathbb{R}^{2}$ be a smooth map defined by

$\Phi(x, y, r)=\left[9(a+\tilde{a})(d+\tilde{d})-(b+\tilde{b})(c+\tilde{c}), 3(a+\tilde{a})(c+\tilde{c})-(b+\tilde{b})^{2}\right](x, y)$, where $r=(\tilde{a}, \tilde{b}, \tilde{c}, \tilde{d}) \in \mathcal{P}_{k}^{4}\left(\mathbb{R}^{2}\right)$. We set $\Phi_{r}(x, y)=\Phi(x, y, r)$.

Lemma 3.3. There exists an open and dense set $\Delta$ of $\mathcal{P}_{k}^{4}\left(\mathbb{R}^{2}\right)$ such that for all $r \in \Delta, 0$ is a regular value of $\Phi_{r}$.

Proof: By Thom transversality lemma, there exists a dense set $\Delta$ of $\mathcal{P}_{k}^{4}\left(\mathbb{R}^{2}\right)$ such that for all $r \in \Delta, 0$ is a regular value of $\Phi_{r}$, that is $\Phi_{r}$ intersect 0 transversally. It is not difficult to show that $H: \mathcal{P}_{k}^{4}\left(\mathbb{R}^{2}\right) \rightarrow$ $C^{\infty}\left(\mathbb{R}^{2}, \mathbb{R}^{2}\right)$ given by

$H(\tilde{a}, \tilde{b}, \tilde{c}, \tilde{d})=\left[9(a+\tilde{a})(d+\tilde{d})-(b+\tilde{b})(c+\tilde{c}), 3(a+\tilde{a})(c+\tilde{c})-(b+\tilde{b})^{2}\right]$ is continuous. Let $r_{0}=\left(a_{0}, b_{0}, c_{0}\right) \in \Delta$. As the set of maps from $\mathbb{R}^{2}$ to $\mathbb{R}^{2}$ which intersect 0 transversally is open, we have that there exists a neighborhood $U$ of $r_{0}$ in $\mathcal{P}_{k}^{4}\left(\mathbb{R}^{2}\right)$ such that for all $r \in U, H(r)=\Phi_{r}$ intersect 0 transversally. The result now follows.

Theorem 3.4. If $a, b, c, d$ are smooth functions, then there exists $a$ good perturbation $F^{t}$ of $(F, 0)$.

Proof: By Lemma 3.3, there exists an open and dense set $\Delta$ of $\mathcal{P}_{k}^{4}\left(\mathbb{R}^{2}\right)$ such that for all $r \in \Delta, 0$ is a regular value of $\Phi_{r}$. It is not difficult to show that there exists a smooth curve $\alpha:(-1,1) \rightarrow \mathcal{P}_{k}^{4}\left(\mathbb{R}^{2}\right)$ such that $\alpha[(-1,1)-\{0\}] \subseteq \Delta$ and $\alpha(0)=0$. Let $\left(a_{t}, b_{t}, c_{t}, d_{t}\right)=(a, b, c, d)+\alpha(t)$. Then, $F^{t}=a_{t} p^{3}+b_{t} p^{2}+c_{t} p+d_{t}=0$ is a good perturbation of the IDE (5).

Definition 3.5. Let $F^{t}$ be a good perturbation of the germ $(F, 0)$ given by (5). Then the cusp index of $(F, 0)$ at 0 is defined by

$$
J(F, 0)=\frac{1}{3} \sum_{i} K_{F^{t}}\left(z_{i}\right)
$$


where $z_{i}$ are non-degenerate singular points of $F^{t}$ of elliptic or hyperbolic cusp type.

The next lemma shows that the index $J(F, 0)$ can be expressed in terms of the coefficients $a, b, c$ and $d$.

Theorem 3.6. Let $(F, 0)$ be the germ of an IDE given by (5). If $F_{p p}(0)=0$ and $O$ is an isolated singular point of $(a, b)$, then

$$
J(F, 0)=\frac{1}{3}\left[\operatorname{Ind}_{0}\left(9 a d-b c, 3 a c-b^{2}\right)-\operatorname{Ind}_{0}(a, b)\right] .
$$

Proof: It follows from Theorem 3.4 that there exists a good perturbation $F^{t}$ of the IDE (5) such that 0 is a regular value of $\left(9 a_{t} d_{t}-b_{t} c_{t}, 3 a_{t} c_{t}-\right.$ $\left.b_{t}^{2}\right), t \neq 0$. Then using Proposition 2.2 in $[7]$ we obtain

$$
\operatorname{Ind}_{0}\left(9 a d-b c, 3 a c-b^{2}\right)=\sum \operatorname{Ind}_{z_{i}}\left(9 a_{t} d_{t}-b_{t} c_{t}, 3 a_{t} c_{t}-b_{t}^{2}\right) .
$$

We denote by $W_{t}=\left(9 a_{t} d_{t}-b_{t} c_{t}, 3 a_{t} c_{t}-b_{t}^{2}\right)$. By Remark 3.2,

$$
\sum_{a_{t}\left(z_{i}\right) \neq 0} \operatorname{Ind}_{z_{i}} W_{t}=K_{F^{t}}\left(z_{i}\right)
$$

As 0 is a regular value of $W_{t}$, we get

$$
\begin{aligned}
\sum_{a_{t}\left(z_{i}\right)=0} \operatorname{Ind}_{z_{i}} W_{t} & =\operatorname{Ind}_{0}\left(a_{t}, b_{t}\right) \\
& =\operatorname{Ind}_{0}(a, b) .
\end{aligned}
$$

The result now follows.

Theorem 3.7. Let $(F, 0)$ and $(G, 0)$ be the germs of IDE's of degree 3 . If $(F, 0)$ and $(G, 0)$ are equivalent, then $J(F, 0)=J(G, 0)$.

Proof: It follows from the hypothesis that there exist a germ of diffeomorphim $h=\left(h_{1}, h_{2}\right):\left(\mathbb{R}^{2}, 0\right) \rightarrow\left(\mathbb{R}^{2}, 0\right)$ and a germ of function $\rho:\left(\mathbb{R}^{2}, 0\right) \rightarrow \mathbb{R}, \rho(0) \neq 0$ such that $G=\rho \cdot(F \circ H)$, where $H(x, y, p)=$ $\left(h(x, y), \frac{h_{2 x}(x, y)+h_{2 y}(x, y) p}{h_{1 x}(x, y)+h_{1 y}(x, y) p}\right)$.

We set $T(x, y, p)=\frac{h_{2 x}(x, y)+h_{2 y}(x, y) p}{h_{1 x}(x, y)+h_{1 y}(x, y) p}$. Then,

$$
\left(\begin{array}{ccc}
\rho & 0 & 0 \\
0 & \rho T_{p} & 0 \\
0 & T_{p p} & \rho T_{p}^{2}
\end{array}\right)\left(\begin{array}{c}
F \circ H \\
F_{p} \circ H \\
F_{p p} \circ H
\end{array}\right)=\left(\begin{array}{c}
G \\
G_{p} \\
G_{p p}
\end{array}\right),
$$

$G_{p p p}=\rho\left[\left(F_{p p p} \circ H\right) T_{p}^{3}+3\left(F_{p p} \circ H\right) T_{p} T_{p p}+\left(F_{p} \circ H\right) T_{p p p}\right]$ and $T_{p}=$ $\frac{\operatorname{det}[d h]}{\left[h_{1 x}+h_{1 y} p\right]^{2}}$. 
By Theorem 3.4, there exists a good perturbation $F^{t}$ of $(F, 0)$. Using equation (9), it is not difficult to show that $G^{t}=\rho \cdot\left(F^{t} \circ H\right)$ is a good perturbation of $(G, 0)$ and

$$
\operatorname{Ind}_{r_{i}}\left(F_{p p p}^{t} F^{t}, F_{p}^{t}, F_{p p}^{t}\right)=\operatorname{Ind}_{q_{i}}\left(G_{p p p}^{t} G^{t}, G_{p}^{t}, G_{p p}^{t}\right),
$$

where $H\left(q_{i}\right)=r_{i}$. The result follows by Remark 3.2 and Definition 3.5.

Corollary 3.8. Let $(F, 0)$ be the germ of an IDE given by (5). If $F_{p p p}(0) \neq 0$ and $F_{p p}(0)=0$, then

$$
J(F, 0)=\frac{1}{3} \operatorname{Ind}_{0}\left(F_{p p p} F, F_{p}, F_{p p}\right) .
$$

Proof: The proof follows by using Theorem 3.6 and Remark 3.2 .

Note that Theorem 2.6 introduce a definition of the index at a fold point of the projection $\pi$ corresponding to a zero of vector field $\xi$. Also from Corollary 3.8 we have a definition of the index at a non-fold singularity of the projection. So we introduce a definition of the index to any germ of IDE, that extends the two definitions given by Theorem 2.6 and Corollary 3.8. Let $U$ be a sufficiently small neighbourhood of 0 in $\mathbb{R}^{2}$.

Definition 3.9. Let $(F, 0)$ be the germ of an IDE and suppose that the number of zeros of the 1 -form $\omega_{\left.\right|_{M}}$ in $(0,0) \times \mathbb{R}$ is finite. Then the index of $(F, 0)$ at 0 is defined by

$$
\begin{aligned}
\operatorname{Ind}_{0} F=\frac{1}{m}\left\{\sum_{i=1}^{n_{0}}\left[\operatorname{Ind}_{s_{i}} \xi+\operatorname{Ind}_{s_{i}}\left(F_{p p} F_{y}, F_{p}, F_{x}+p F_{y}\right)\right]\right. & \\
& \left.+\sum_{i=1}^{n_{1}} \operatorname{Ind}_{s_{i}}\left(F_{p p p} F, F_{p}, F_{p p}\right)\right\},
\end{aligned}
$$

where $s_{i}=\left(0,0, p_{i}\right)$ and $m$ is the maximum number of integral curves that pass through $q \in U$.

Theorem 3.10. Let $(F, 0)$ be the germ of an IDE of degree $n$. Then,

(1) If $n=2, F_{p p}(0) \neq 0$ and 0 is a zero of $\xi$, then $\operatorname{Ind}_{0} F=I(F, 0)$.

(2) If $n=3, F_{p p}(0)=0, F_{p p p}(0) \neq 0$ and 0 is not a zero of $\xi$, then $\operatorname{Ind}_{0} F=J(F, 0)$.

Proof: The first statement follows from Theorem 2.6. Part (2) follows from Corollary 3.8. 
Theorem 3.11. Let $(F, 0)$ and $(G, 0)$ be the germs of IDE's and suppose that the number of zeros of the 1 -form $\omega_{\left.\right|_{M}}$ in $(0,0) \times \mathbb{R}$ is finite. If $(F, 0)$ and $(G, 0)$ are equivalent, then $\operatorname{Ind}_{0} F=\operatorname{Ind}_{0} G$.

Proof: From [11] and Lemma 6.1 in [5], we obtain that

$$
\operatorname{Ind}_{s_{i}} \xi=\operatorname{Ind}_{s_{i}}\left(F F_{y}, F_{p}, F_{x}+p F_{y}\right) .
$$

It follows from the hypothesis that there exist a germ of diffeomorphim $h=\left(h_{1}, h_{2}\right):\left(\mathbb{R}^{2}, 0\right) \rightarrow\left(\mathbb{R}^{2}, 0\right)$ and a germ of function $\rho:\left(\mathbb{R}^{2}, 0\right) \rightarrow \mathbb{R}, \rho(0) \neq 0$ such that $G=\rho \cdot F \circ H$, where $H(x, y, p)=$ $\left(h(x, y), \frac{h_{2 x}(x, y)+h_{2 y}(x, y) p}{h_{1 x}(x, y)+h_{1 y}(x, y) p}\right)$.

We set $T(x, y, p)=\frac{h_{2 x}(x, y)+h_{2 y}(x, y) p}{h_{1 x}(x, y)+h_{1 y}(x, y) p}$, and to simplify the arguments, we take $\rho=1$. Then $\operatorname{det}[d H]=\operatorname{det}[d h] \cdot T_{p}$ and

$$
\left(\begin{array}{ccc}
S & G T_{y} & h_{1 y} G \\
0 & T_{p} & 0 \\
0 & S_{1} & S_{2}
\end{array}\right)\left(\begin{array}{c}
\left(F_{y} F\right) \circ H \\
F_{p} \circ H \\
\left(F_{x}+p F_{y}\right) \circ H
\end{array}\right)=\left(\begin{array}{c}
G_{y} G \\
G_{p} \\
G_{x}+p G_{y}
\end{array}\right)
$$

where $S=\frac{\operatorname{det}[d h]}{h_{1 x}+p h_{1 y}}, S_{1}=T_{x}+p T_{y}$ and $S_{2}=h_{1 x}+p h_{1 y}$. Using equation (10), we get

$$
\operatorname{Ind}_{s_{i}}\left(F_{y} F, F_{p}, F_{x}+p F_{y}\right)=\operatorname{Ind}_{r_{i}}\left(G_{y} G, G_{p}, G_{x}+p G_{y}\right),
$$

where $s_{i}=H\left(r_{i}\right)$. Following the same arguments as above we show that

$$
\begin{aligned}
\operatorname{Ind}_{s_{i}}\left(F_{p p} F_{y}, F_{p}, F_{x}+p F_{y}\right) & =\operatorname{Ind}_{r_{i}}\left(G_{p p} G, G_{y}, G_{x}+p G_{y}\right) \\
\operatorname{Ind}_{s_{i}}\left(F_{p p p} F, F_{p}, F_{p p}\right) & =\operatorname{Ind}_{r_{i}}\left(G_{p p p} G, G_{p}, G_{p p}\right),
\end{aligned}
$$

and the result follows.

\section{Index of a differential $n$-form}

Let $M$ be a $C^{\infty}$-compact, connected, oriented, 2-dimensional surface. A (symmetric) differential $n$-form on $M$ is a differentiable section of the symmetric tensor fiber bundle $S^{n}\left(T^{*} M\right)$. Let $\omega$ be a differential $n$-form on $M$. An integral curve of $\omega$ is a smooth curve $\alpha:(-1,1) \rightarrow M$ such that $\omega(\alpha(t))\left(\alpha^{\prime}(t)\right)=0$

We denote by $\mathbb{H}^{m}$ the set of points $p$ in $M$ such that the subset $\omega(p)^{-1}(0)$ is the union of $m$ transversal lines. Through every point $p$ of $\mathbb{H}^{m}$ pass $m$ transverse integral curves of $\omega$. Under the orientability hypothesis imposed on $M$, the differential $n$-form $\omega$ defines $m$ line fields $L_{\omega, 1}, \ldots, L_{\omega, m}$ on $\mathbb{H}^{m}$ such that the integral curves of $L_{\omega, i}$ correspond to integral curves of $\omega$. 
Let $P M$ be the projectivized tangent bundle of $M$, and $\mathcal{H} \subset P M$ the set of points $(p,[v])$ such that $\omega(p)(v)=0$. Let $\pi: P M \rightarrow M$ be the natural projection given by $\pi(p,[v])=p$. Then, the restriction of the projection $\pi$ to $\mathcal{H}$ covers the closure of $\mathbb{H}=\bigcup_{i=1}^{n} \mathbb{H}^{i}$. Lifting to $\mathcal{H}$ the line fields $L_{\omega, 1}, \ldots, L_{\omega, n}$ define a single line field $L$ on $\pi^{-1}(\mathbb{H})$ which, generically, uniquely extends to a smooth line field $L$ defined on the whole $\mathcal{H}$.

Let $h: U \rightarrow \mathbb{R}^{2}$ be a local chart of $M$ at $p_{0}$ such that $h\left(p_{0}\right)=0$. Then, the pull-back of $\omega$ defines an IDE of degree $n$ given by

(11) $h^{*}(\omega)(x, y, p)=a_{0}(x, y) p^{n}+a_{1}(x, y) p^{n-1}+\cdots+a_{n}(x, y)=0$

where $p=\frac{d y}{d x}$ and $a_{i}: \mathbb{R}^{2} \rightarrow \mathbb{R}$ are smooth functions. We say that $p_{0} \in M$ is a singular point of $\omega$ if 0 is a singular point of $h^{*}(\omega)$.

Definition 4.1. Let $p_{0}$ be a singular point of $\omega$. Then, the index of $\omega$ at $p_{0}$ is defined by $\operatorname{Ind}_{p_{0}} \omega=\operatorname{Ind}_{0} h^{*}(\omega)$.

The next lemma shows that the index is independent of the choice of a local chart of $M$.

Lemma 4.2. Let $p_{0}$ be a singular point of $\omega$ and $h_{1}: V \rightarrow \mathbb{R}^{2}$ a local chart of $M$ at $p_{0}$ such that $h_{1}\left(p_{0}\right)=0$. Then, $\operatorname{Ind}_{0} h^{*}(\omega)=\operatorname{Ind}_{0} h_{1}^{*}(\omega)$.

Proof: Note that $h^{*}(\omega)(z)[u]=\omega\left(h^{-1}(z)\right)\left[d h_{z}^{-1}(u)\right]$, where $z, u \in \mathbb{R}^{2}$. Then, the map $h_{1} \circ h^{-1}: \mathbb{R}^{2} \rightarrow \mathbb{R}^{2}$ sends integral curves of $h^{*}(\omega)$ to integral curves of $h_{1}^{*}(\omega)$. Therefore, by Theorem 3.11 the result follows.

We denote by $(\omega, p)$ the germ of the differential $n$-form $\omega$ at a singular point $p$. Let $\left(\omega_{1}, p_{1}\right),\left(\omega_{2}, p_{2}\right)$ be two germs of differential $n$-forms defined on 2-dimensional surfaces $M, N$, respectively. We say that they are equivalent if there exists a germ of diffeomorphism $k:\left(M, p_{1}\right) \rightarrow\left(N, p_{2}\right)$ that sends integral curves of $\left(\omega_{1}, p_{1}\right)$ to integral curves of $\left(\omega_{2}, p_{2}\right)$. We denote by $\overline{\mathbb{H}}^{m}$ the closure of $\mathbb{H}^{m}$.

Theorem 4.3. Suppose that $\mathcal{H}$ is a smooth surface, $\pi_{\mid \mathcal{H}}$ is a stable map without cusp and transverse folds. If all the zeros of the line field $L$ belong to $\pi_{\mid \mathcal{H}}^{-1}\left(\overline{\mathbb{H}}^{m}\right)$, then $\chi(\mathcal{H})=m \cdot \chi(\pi(\mathcal{H}))$.

Proof: Since by hypothesis there exist $m$ line fields $L_{\omega, 1}, \ldots, L_{\omega, m}$ on $\mathbb{H}^{m}$ which are linearly independent, and $\chi\left(L_{\omega, i}\left(\overline{\mathbb{H}}^{m}\right) \cap L_{\omega, j}\left(\overline{\mathbb{H}}^{m}\right)\right)=0, i \neq j$. As $\pi_{\mid \mathcal{H}}^{-1}\left(\overline{\mathbb{H}}^{m}\right)=\bigcup_{i=1}^{m} L_{\omega, i}\left(\overline{\mathbb{H}}^{m}\right)$, it follows that $\chi\left(\pi_{\left.\right|_{\mathcal{H}}}^{-1}\left(\overline{\mathbb{H}}^{m}\right)\right)=m \cdot \chi\left(\overline{\mathbb{H}}^{m}\right)$. Analogously one proves that $\chi\left(\pi_{\left.\right|_{\mathcal{H}}}^{-1}\left(\overline{\mathbb{H}}^{s}\right)\right)=s \cdot \chi\left(\overline{\mathbb{H}}^{s}\right), s \neq m$. As the restriction of the line field $L$ to the surface $\pi_{\left.\right|_{\mathcal{H}}}^{-1}\left(\overline{\mathbb{H}}^{s}\right)$ has no zeros, we 
have that $\chi\left(\pi_{\mid \mathcal{H}}^{-1}\left(\overline{\mathbb{H}}^{s}\right)\right)=0$ and $\chi\left(\overline{\mathbb{H}}^{s}\right)=0$. Since $\mathcal{H}=\pi_{\mid \mathcal{H}}^{-1}\left(\overline{\mathbb{H}}^{m}\right) \cup$ $\left(\bigcup_{i \neq m} \pi_{\mid \mathcal{H}}^{-1}\left(\overline{\mathbb{H}}^{i}\right)\right)$ and $\pi(\mathcal{H})=\overline{\mathbb{H}}^{m} \cup\left(\bigcup_{i \neq m} \overline{\mathbb{H}}^{i}\right)$, the result follows.

Theorem 4.4. Suppose that $\mathcal{H}$ is a smooth surface, $\pi_{\mid \mathcal{H}}$ is a stable map without cusp and transverse folds. If $\mathbb{H} \neq \emptyset$ and $n$ is odd, then

$$
\chi(M)=\sum_{i=1}^{s} \operatorname{Ind}_{p_{i}} \omega,
$$

where $p_{1}, \ldots, p_{s}$ are the singular points of $\omega$.

Proof: Let $q_{i 1}, \ldots, q_{i r}$ be the zeros of the line field $L$ belong to $\pi_{\mid \mathcal{H}}^{-1}\left(\overline{\mathbb{H}}^{i}\right)$. Then using the Poincaré-Hopf Theorem we obtain

$$
\chi\left(\pi_{\mid \mathcal{H}}^{-1}\left(\overline{\mathbb{H}}^{i}\right)\right)=\sum_{j=1}^{r} \operatorname{Ind}_{q_{i j}} L .
$$

By Theorem 4.3 and Definition 3.9,

$$
\begin{aligned}
\chi\left(\overline{\mathbb{H}}^{i}\right) & =\frac{1}{i} \cdot \sum_{j=1}^{r} \operatorname{Ind}_{q_{i j}} L \\
& =\sum_{j=1}^{r} \operatorname{Ind}_{p_{i j}} \omega,
\end{aligned}
$$

where $p_{i j}=\pi\left(q_{i j}\right)$. Since $M=\bigcup_{i=1}^{n} \overline{\mathbb{H}}^{i}$, the result follows.

\section{Applications}

Let $M$ be a 2-dimensional smooth surface in $\mathbb{R}^{5}$, and denote by $T M$ and $N M$ its tangent and normal bundles. The contact of the surface with 4-dimensional planes is measured by the singularities of the height function

$$
H: M \times S^{4} \longrightarrow \mathbb{R} \times S^{4}
$$

given by $H(q, v)=\left(h_{v}(q), v\right)$, where $h_{v}(q)=\langle q, v\rangle$. A height function $h_{v}$ has a singularity at $q \in M$ if and only if $v \in N_{q} M$. It follows from Looijenga's theorem [2] that $h_{v}$ has generically a singularity of type $A_{k \leq 5}$, $D_{4}^{ \pm}$or $D_{5}$ (see [1] for notation).

A direction $v \in N_{q} M$ is said to be degenerate if $q$ is a non-stable singularity of $h_{v}$. In this case, the kernel of the Hessian of $h_{v}, \operatorname{ker}\left(\operatorname{Hess}\left(h_{v}\right)(q)\right)$, contains non zero vectors. Any direction $u \in \operatorname{ker}\left(\operatorname{Hess}\left(h_{v}\right)(q)\right)$ is called a contact direction associated to $v$.

A unit vector $v \in N_{q} M$ is called a binormal direction if $h_{v}$ has a singularity of type $A_{3}$ or worse at $q$. 
Definition 5.1 ([17]). Let $q \in M$ and $v \in N_{q} M$ be a binormal direction. An asymptotic direction at $q$ is any contact direction associated to $v$.

Theorem $5.2([\mathbf{1 8}])$. There is at least one and at most five asymptotic curves passing through any point on a generic immersed surface in $\mathbb{R}^{5}$. These curves are solutions of the implicit differential equation $A_{0} d y^{5}+A_{1} d y^{4} d x+A_{2} d y^{3} d x^{2}+A_{3} d y^{2} d x^{3}+A_{4} d y d x^{4}+A_{5} d x^{5}=0$, where the coefficients $A_{i}, i=0,1,2,3,4,5$ depend on the coefficients of the second fundamental form and their first order partial derivatives.

For a generic surface, at least one of the coefficients in the Theorem 5.2 is not zero at any point $q \in M$. We then set $p=\frac{d y}{d x}$ (as $d x=0$ is not solution of the equation) so that the equation of the asymptotic curves in a neighbourhood $U$ of $q$ is an IDE of degree 5 in the form

$$
F(x, y, p)=A_{0} p^{5}+A_{1} p^{4}+A_{2} p^{3}+A_{3} p^{2}+A_{4} p+A_{5}=0 .
$$

It follows that there exists a differential 5-form $\eta$ on $M$ such that $\eta=F$ in $U$. The discriminant of the asymptotic curves $\Delta_{\eta}$ is given by the discriminant of the IDE (12).

Theorem 5.3. Let $M$ be a closed orientable surface generically immersed in $\mathbb{R}^{5}$. Then

$$
\chi(M)=\sum_{i=1}^{s} \operatorname{Ind}_{q_{i}} \eta,
$$

where $q_{1}, \ldots, q_{s}$ are the singular points of $\eta$.

Proof: Since $\Delta_{\eta}$ is a smooth curve, the result then follows by Theorem 4.4.

A folded singularity of the asymptotic curves is a folded-singularity of the IDE (12). The following result also appears in [18].

Corollary 5.4. Let $M$ be a closed orientable surface generically immersed in $\mathbb{R}^{5}$ with $\chi(M) \neq 0$. Then $\Delta_{\eta}$ is not empty and the asymptotic curves have folded singularities.

Proof: The proof follows by using Theorem 5.3.

\section{References}

[1] V. I. Arnol'D, S. M. Gusě̌n-Zade, And A. N. Varchenko, "Singularities of differentiable maps", Vol. I. The classification of critical points, caustics and wave fronts, Translated from the Russian by Ian Porteous and Mark Reynolds, Monographs in Mathematics 82, Birkhäuser Boston, Inc., Boston, MA, 1985. 
[2] TH. BRÖCKER, "Differentiable germs and catastrophes", Translated from the German, last chapter and bibliography by L. Lander, London Mathematical Society Lecture Note Series 17, Cambridge University Press, Cambridge-New York-Melbourne, 1975.

[3] J. W. BRUCE AND F. TARI, On the multiplicity of implicit differential equations, J. Differential Equations 148(1) (1998), 122-147.

[4] J. W. BRuCE AND F. TARI, Dupin indicatrices and families of curve congruences, Trans. Amer. Math. Soc. 357(1) (2005), 267-285 (electronic).

[5] L. S. Challapa, Invariants of binary differential equations, J. Dyn. Control Syst. 15(2) (2009), 157-176.

[6] L. S. Challapa, Index of quadratic differential forms, in: "Real and complex singularities", Contemp. Math. 459, Amer. Math. Soc., Providence, RI, 2008, pp. 179-193.

[7] A. Cima, A. Gasull, and J. Torregrosa, On the relation between index and multiplicity, J. London Math. Soc. (2) 57(3) (1998), 757-768.

[8] L. DARA, Singularités génériques des équations différentielles multiformes, Bol. Soc. Brasil. Mat. 6(2) (1975), 95-128.

[9] A. A. DAvydov, The normal form of a differential equation, that is not solved with respect to the derivative, in the neighborhood of its singular point, (Russian), Funktsional. Anal. i Prilozhen. 19(2) (1985), 1-10, 96; English translation: Functional Anal. Appl. 19(2) (1985), 81-89.

[10] W. EBeling and S. M. Guseřn-Zade, On the index of a vector field at an isolated singularity, in: "The Arnoldfest" (Toronto, ON, 1997), Fields Inst. Commun. 24, Amer. Math. Soc., Providence, RI, 1999, pp. 141-152.

[11] W. EBeling And S. M. Guseřn-Zade, Indices of 1-forms on an isolated complete intersection singularity, Dedicated to Vladimir I. Arnold on the occasion of his 65 th birthday, Mosc. Math. J. 3(2) (2003), 439-455, 742-743.

[12] T. Fukui And J. J. Nuño-Ballesteros, Isolated singularities of binary differential equations of degree $n$, Preprint, available at http://www.uv.es/nuno/Preprints.htm.

[13] R. Garcia, C. Gutierrez, and J. Sotomayor, Structural stability of asymptotic lines on surfaces immersed in $\mathbb{R}^{3}$, Bull. Sci. Math. 123(8) (1999), 599-622.

[14] V. Guíñez, Positive quadratic differential forms and foliations with singularities on surfaces, Trans. Amer. Math. Soc. 309(2) (1988), 477-502. 
[15] A. G. KuZ'Min, "Nonclassical equations of mixed type and their applications in gas dynamics", Translated and revised from the Russian by the author, International Series of Numerical Mathematics 109, Birkhäuser Verlag, Basel, 1992.

[16] J. W. MilnoR, "Topology from the differentiable viewpoint", Based on notes by David W. Weaver, The University Press of Virginia, Charlottesville, Va., 1965.

[17] D. K. H. Mochida, M. C. Romero-Fuster, And M. A. S. RUAS, Inflection points and nonsingular embeddings of surfaces in $\mathbb{R}^{5}$, Rocky Mountain J. Math. 33(3) (2003), 995-1009.

[18] M. C. Romero-Fuster, M. A. S. Ruas, and F. Tari, Asymptotic curves on surfaces in $\mathbb{R}^{5}$, Commun. Contemp. Math. 10(3) (2008), 309-335.

L. S. Challapa:

Universidade Federal de Paraíba - DM

Cidade Universitária

58051-900 João Pessoa - PB

Brazil

E-mail address: challapa@mat.ufpb.br

M. A. S. Ruas:

Universidade de São Paulo - ICMC

Caixa Postal 668

13560-970, São Carlos - SP

Brazil

E-mail address: maasruas@icmc.usp.br

Primera versió rebuda el 24 d'octubre de 2008, darrera versió rebuda el 14 d'abril de 2009. 